Aus der 1. Chirurgischen Abteilung des Krankenhauses am Urban in Berlin. (Direktor: Geh.-Rat Körte.)

\title{
Ueber Blutreïnfusion bei 24 Fällen von Graviditas
} extrauterina rupta ${ }^{2}$.

Von Dr. Bernhard Töpler, Assistenzarzt.

Nachdem Thies 1914 die Eigenblutreïnfusion bei Graviditas tubaria rupta empfohlen, ist sie auch von den Chirurgen aufgenommen und während des Krieges bei großen Blutungen in die Bauch- und Brusthöhle, bei SçhuBverletzungen, Milz- und Leberrupturen mit gutem

1) C. Kl e in, Beitrag zur Pneumoniebehandlung mit hohen Kampferdosen; Kampfervergiftuń ; $M$. Kl. 1920 Nr. 37. - ${ }^{2}$ ) Vorgetragen in der Sitzung der Berliner Gesellschaft fur Chiturgie am 24. X. 1921 : 
Erfolge angewandt worden. Ihr Hauptanwendungsgebiet ist die ge platzte $\mathrm{Bauch}$ ählenschwangerschaft geblieben, und zwar vor allem deshalb, weil die bisherigen Behandlungsmethoden in einer Anzahl von Fällen den Verblutungstod nicht aufzulialten vermögen. Nach einer Statistik Lichtensteins aus dem Jahre 1919 starben von den in der Literatur angegebenen Fällen von geplatzter Bauchhöhlenschwangerschaft $12 \%$ an innerer Blutung; von den an unser Abteilung operierten 71 Fällen der Jahre 1910-1918 starben 5,7\% an dem Blutverlust, obgleich baldmöglichst operiert und ausgiebig physiologische NaCl-Lösung infundiert wurde. Um auch solche Frauen zu retten, wurde das von $\mathrm{T}$ h i e s angegebene Verfahren der Reïnfusion von Eigenblut von uns aufgenommen und nach dem Kriege in 24 Fällen angewandt.

ธei der Auswahl der für die Blütreïnfusion geeigneten Fälle wurden die von Lichtenstein aufgestellten Vorbedingungen erfüllt. Es kamen daher nur frische Rupturen mit lelsenstähigem Blut in Frage, das nach Vorgeschichte und Operationsbefund als nicht toxisch 1und nicht infiziert angesehen werden konnte. Fälle mit Hämatozelenbilduııg wurden nicht herangezogen, dagegen 3 Fälle, bei denen kleinere Blutungen vorangegangen, die aber durch eine neue abundante Blutung in Lebensgefahr geraten waren. Bei einer Frau, die besinnungslos in ihrer Wohnung, wo sie schon mehrere Tage gelegen haben soll, aufgefunden wurde, wurde die Reïnfusion versucht, der Fall selbst aber aus obigen Guründen in die Statistik nicht mit einbezogen.

In beiden Chirurgischen Abteilungen des Krankenhauses am Urban wurden die Rupturen nach gleichen Gesichtspunkten behandelt. Unter den 40 seit Kriegsschluß zur Operation gebrachten Fällen (24 der I., 16 der II. Chir. Abt.) wurde aber nochmals eine Auswahl getroffen derart, daß die leichteren und mittelschweren Fälle, bei denen erfahrn111gsgemäß die einfache $0,9 \%$ ige NaCl-Lösung zum Blutersatz ansreicnt, im allgemeinen nicht mit Blutreïnfusion behandelt wurden. Die Reïnfusion blieb vielmehr auf 24 Fälle beschränkt, die klinisch einen schwer kranken Eindruck machten, die bei hochgradiger Anämie, kiulm fühlbarem Puls, Atemnòt und Kollaps dem Verblutungstod nahe waren.

Bei der Begutachtung der Fälle war vor allem die klinische Beobachtung maligebend, da die Untersuchung des Blutdrucks, Hämoglobingehaltes und der Zahl der roten und weißen Blutkörperchen nicht regelmäßig möglich war.

Die Mehrzahl der Patienten stand im Alter von 26 bis 33 Jahren. 16 voll ihnen waren schon vorher uterin schwanger gewesen. Die Ruptur erfolgte meistens in der 6 . Schwangerschaftswoche. In 19 Fällen fand die Operation in den ersten 24 Stunden statt, in 2 Fällen am 2. Tag, $3 \mathrm{~F}$ rauen gaben eine längere Rupturdauer an. Die Laparotonnie ergab in 22 Fällen Tubenschwangerschaft mit äußerem Fruchtkapselaufbruch, in 2 Fällen Bauchhöhlenschwangerschaft. Die Diagnose wurde stets vor der Operation gestellt.

Die Technik der Infusion gestaltet sich derart, daß nach Eröffnung des Peritoneums die rupturierte Tube abgeklemmt wird. Darauf wird mit dem Schöpflöffel das freie Blut der Bauchhöhle, einschließlich Koagula, ausgeschöpft, durch 8fache Mullschicht geseiht und in einem großen Erlmeyerkolben aufgefangen. Dort wird das Blut mit physiologischer NaCl-Lösung verdünnt und im Wasserbade so lange auf Körperwärme gehalten, bis es durch eine Kanüle, die ein Assistent inzwischen in die Vena mediana cubiti einbindet, einfließen kann. Die Reinfusion erfolgt, während der Operateur die geborstene Tube versorgt und die Bauchwunde schlieBt. Das ausgeschöpfte Blut wird weder defibriniert noch mit Natr-citric.-Lösung vermischt. Das Mischungsverhältnis von Blut und $\mathrm{NaCl}$-Lösung ist etwa 1:1. Aus $1 / 2 \mathrm{~m}$ Höhe fließt das im Kolben verdünnte Blut durch die Körtesche Kanüle in 10-40 Minuten ab. Die Lichtensteinsche Ventilspritze wurde nicht verwandt. In sämtlichen Fällen gelang die Infusion ohne große Schwierigkeit. Es gelang stets, die Kanüle in die Vena mediana cubiti eiluzubinden, die von Doe de rl e i n zur Infusion vorgeschlagene Vena spermatica interna oder eine größere Netzvene brauchten nicht benutzt zu werden. Eine Verlegung der Kanüle durch Gerinnsel kam nicht vor. Die vorgesehene Blutmenge floß immer ohne größeren Rest $a b$, die unvermittelte Blutmenge betrug im Durchschnitt $520 \mathrm{ccm}$, die größte Menge 900 , die kleinste $150 \mathrm{ccm}$.

Die Zufuhr dieser Blutmengen haben sämtliche 24 Patientinnen gut vertragen. Krankhafte Erscheinungen, wie Somnolenz, Unruhe, Ilkterus, die Opitz auf die Intoxikation durch die Zerfallsprodukte der reînfundierten roten Blutkörperchen zurückführt, sind nicht eingetreten. Schüttelfrost, Dyspnoe, Zyanose, von A rnim als Folgen kapillärer Embolien gedeutet, haben wir nicht gesehen. Auch ${ }^{-B} \mathrm{um} \mathrm{m} \mathrm{s}$ Beobachtungen von Frost mit hohem Temperaturanstieg konnten wir nicht machen. Hämoglobinurie; woran Schweitzer eine Frau sterben sah, trat bei uns nicht auf. Die Operierten zeigten als einziges abnormes Merkmal erhöhte, meist subfebrile Temperaturen, wie sie nach Laparotomie infolge geborstener Tubenschwangerschaft liicht selten sind, die wohl aber in der Blutreinfusion keine Ursache haben. Peritonitische Erscheinıngen sind nicht aufgetreten. Die Beobachtung, daß nach Gefäßversorgung der Tube die Patientinnen sich sehr bald erholen, haben wir bei sehr vielen, auch nicht reïnfundierten Frauen gemacht. Bei letzteren war das Aufblühen aber ganz beson1. ders deutlich, da das erste Krankheitsbild meist schwerer war und die Kranken trotzdem bald die Leichenblässe ihrer Haut verloren, fühlbaren Puls und ruhige Atmung bekamen. Am nächsten Tage zeigten die Patientinnen frische Hautfarbe, gesteigerten Blutdruck, zunehmendes Kräftegefühl. Sämtliche 24 mit Reïnfusion behandelten
Fälle kol111ten nach kurzer Zeit geheilt entlassen werden. Die Behandlungsdauer betrug im Durchschnitt 20 Tage, sie war kürzer als die der nicht reinfundierten Nachkriegsfälle und als die Durchschnittskrankheitsdauer der vor dem Kriege behandelten. Zu der Menge des reïnfundierten Blutes steht die Krankheitsdauer in keinem bestimmten Verhältnis, etwa in der Weise, daß erhöhte Blutzufuhr schnellere Herstellung herbeiführe.

Zusammenfassung: Bei 24 Fällen schwerster akuter Anämie infolge Graviditas extrauterina rupta ist die Eigenblutreïnfusion mit gutem Erfolge angewandt worden: sämtliche Patientinnen wurden geheilt, Komplikationen sind nicht eingetreten. Im Einzelfall ist oft die Wirkung der Eigenblutreïnfusion von der Zufuhr der NaCl-Lösung kaum zu unterscheiden, bei Beobachtung einer größeren Anzahl vor Fällen kommt man alser doch zu der Ueberzeugung, daß die Eigenblutreïnfusion geeignet ist, noch manchen Fall zu retten, bei dem die $\mathrm{NaCl}$-Lösung nicht mehr ausreichen würde. 